\title{
Chromosomal stasis versus plasmid plasticity in aphid endosymbiont Buchnera aphidicola
}

\author{
A Latorre, R Gil, FJ Silva and A Moya \\ Institut Cavanilles de Biodiversitat i Biologia Evolutiva, Universitat de València, Apartado de Correos 2085, 46071 Valencia, Spain
}

\begin{abstract}
The study of three genomes of the aphid endosymbiont Buchnera aphidicola has revealed an extraordinary stasis: conservation of gene order and genetic composition of the chromosome, while the chromosome size and number of genes has reduced. The reduction in genome size appears to be ongoing since some lineages we now know to have even smaller chromosomes than the first $B$. aphidicola analysed. The current sequencing by our group of one of these smaller genomes with an estimated size of $450 \mathrm{~kb}$, and its comparison with the other three available genomes provide insights into the nature of processes involved in shrinkage. We discuss whether $B$. aphidicola might be driven to extinction and be replaced by secondary aphid endosymbionts. In some lineages, genes encoding key enzymes in
\end{abstract}

the pathways leading to tryptophan and leucine biosynthesis (trpEG and leuABCD, respectively) are located on plasmids, rather than the main chromosome. In contrast to the stasis of the main chromosome, plasmid genes have frequently been transferred to the main chromosome and undergone other gene rearrangements. We propose a two-step scenario to explain these contrasting modes of evolution. Essential genes may have escaped regulation by moving to plasmids in a moving $B$. aphidicola ancestor. $B$. aphidicola became polyploidy at a given stage of its evolution and plasmid genes have been transferred to the main chromosome through several independent events.

Heredity (2005) 95, 339-347. doi:10.1038/sj.hdy.6800716; published online 24 August 2005

Keywords: comparative genomics; gene order; leucine and tryptophan plasmids; endosymbionts

\section{Introduction}

Contrary to most prokaryotes, eukaryotes have rather limited metabolic capabilities, and hence, symbiosis has provided an evolutionary strategy through which eukaryotes gain access to a wider range of metabolic resources. Insects are particularly prone to symbiotic associations with microorganisms (Buchner, 1965). It has been estimated that at least 15-20\% of insects live in such symbiotic relationships, allowing them to explore a great variety of ecological niches. Insects that live in close association with bacteria are characterized, in general, by feeding on unbalanced diets, poor in essential nutrients such as amino acids, sterols or vitamins, which are provided by the symbionts (Sasaki et al, 1991; Douglas, 1998; Baumann et al, 2000). Such is the case of members of the orders Homoptera (aphids, whiteflies, mealybugs, psylids and cicadas), Blattaria (cockroaches) and Coleoptera (weevils). Insect endosymbionts live in a very closed environment, inside specialised host cells called bacteriocytes, which may form an organ-like structure called bacteriome, in the body cavity of the insects. The association is mutualistic and obligate for both partners: the bacteria cannot be cultured outside the host, whereas the host needs the bacteria for normal growth and reproduction.

Correspondence: A Latorre, Institut Cavanilles de Biodiversitat i Biologia Evolutiva, Universitat de València, Apartado de Correos 2085, 46071 Valencia, Spain. E-mail: amparo.latorre@uv.es

Received 26 November 2004; accepted 16 June 2005; published online 24 August 2005
In the last few years, five complete genomes of insect endosymbionts have been fully sequenced, all of them belonging to the $\gamma$-Proteobacteria: three strains of Buchnera aphidicola (Shigenobu et al, 2000; Tamas et al, 2002; van Ham et al, 2003), Wigglesworthia glossinidia (Akman et al, 2002) and Blochmannia floridanus (Gil et al, 2003), the primary endosymbionts of aphids, tsetse flies and carpenter ants, respectively. These genomes have a size ranging from 618 to $706 \mathrm{~kb}$, and contain 545-661 genes, thus revealing a dramatic process of genome and gene number reduction when compared to close freeliving relatives such as Escherichia coli (E. coli $\mathrm{K} 12$ has a $4639 \mathrm{~kb}$ genome, containing 4289 genes) (Blattner et al, 1997). The comparison of the genes present in these bacteria confirmed the nutritional role of the symbioses, since all of them contain species-specific genes involved in the supply of products that are needed by their particular hosts, in addition to a common set of genes necessary for intracellular life (Gil et al, 2003; Klasson and Andersson, 2004).

Aphids are plant phloem-feeding insects, whose diet is deficient in essential amino acids, which are provided by the primary endosymbiont $B$. aphidicola. The association between aphids and B. aphidicola is very ancient, and the congruence between the phylogenetic trees of hosts and symbionts indicates a unique infection event about 84-164 million years ago, followed by the coevolution of both partners (Moran et al, 1993; von Dohlen and Moran, 2000). The genus Buchnera contains one species, $B$. aphidicola, which designates all primary endosymbionts present in the different aphid species (Munson et al, 1991). B. aphidicola is maternally inherited by infection of 
the eggs or embryos at the blastoderm stage (Buchner, 1965). For the last few decades, many studies have been carried out regarding the biology of aphids, B. aphidicola, and the aphid-B. aphidicola association (revised in Douglas, 1998; Baumann et al, 2000; Latorre et al, 2003), as it is considered an excellent model to gain insights into the extreme evolutionary reductive process that takes place when bacterial lineages make the transition from an independent lifestyle to a permanent association with the host.

In addition to its drastic genome reduction and the proliferation of plasmids involved in the biosynthesis of the amino acids leucine and tryptophan (see below), $B$. aphidicola has undergone many other important molecular and biochemical changes, when compared to its freeliving relatives. These changes can be characterised by the following features: amplification of the number of copies of the main chromosome per cell, an almost total absence of recombination, an increase in the rate of nucleotide substitution, high $\mathrm{A}+\mathrm{T}$ content, an accumulation of deleterious mutations and loss of codon bias (Moran, 1996; Clark et al, 1999; Komaki and Ishikawa, 1999; Baumann et al, 2000; Moya et al, 2002).

\section{Gene order and gene content in the B. aphidicola chromosome}

In recent years, many studies performed on both symbionts and parasites have shown that, once the intracellular lifestyle is established, a massive process of genome degradation occurs, resulting in the inactivation and loss of many genes, reflecting the relaxation of selection to maintain genes that are rendered superfluous in the constant and rich environment provided by the host (Andersson and Kurland, 1998; Moran and Mira, 2001; Silva et al, 2001; Moran, 2002; Gómez-Valero et al, 2004a). The genomes of three B. aphidicola obtained from different aphid species are currently available: $B$. aphidicola (BAp) from Acyrthosiphon pisum, B. aphidicola (BSg) from Schizaphis graminum, and B. aphidicola (BBp) from Baizongia pistaciae (Table 1) (Shigenobu et al, 2000; Tamas et al, 2002; van Ham et al, 2003). In addition, we are currently sequencing the genome of $B$. aphidicola (BCc), from Cinara cedri (Pérez-Brocal et al, 2005). The comparison of the gene content and gene order in these four $B$. aphidicola strains associated with aphids from three different subfamilies can give some clues about specific adaptations of the bacteria to the particular environment provided by their respective hosts, leading to different structural changes in their genomes.

\section{Gene-order fossil in B. aphidicola}

The sequencing of the first $B$. aphidicola genome from the aphid A. pisum (BAp) (Shigenobu et al, 2000), and its comparison to the genomes of E. coli (Blattner et al, 1997) and Vibrio cholerae (Heidelberg et al, 2000), two free-living bacteria closely related to $B$. aphidicola, indicated that the endosymbiont has not only experienced substantial genome reduction but also many chromosomal rearrangements since the divergence of these species, the most frequent ones being the inversions around the origin and terminus of replication (Silva et al, 2001).

Soon after, a second B. aphidicola genome was sequenced from S. graminum (BSg) (Tamas et al, 2002), an aphid belonging to the same subfamily as $A$. pisum (Aphidinae), but to a different tribe (Aphidini for S. graminum, and Macrosiphini for A. pisum) (see Table 2). The estimated divergence time between these tribes is 50-70 million years (Clark et al, 1999). The comparison between both genomes revealed an extreme degree of conservation, without either chromosomal rearrangements (translocations, inversions or duplications), or gene acquisition by horizontal gene transfer, thus being the most extreme case of genome stability to date (Tamas et al, 2002). Comparison with a third strain, B. aphidicola $\mathrm{BBp}$, associated with the aphid B. pistaciae (subfamily Pemphiginae), confirmed that the genomic architecture is extremely similar. Near-perfect gene-order conservation was found, with only four minor rearrangements (two inversions and two translocations involving the leucine and tryptophan plasmid-contained genes) relative to the BAp and BSg strains (van Ham et al, 2003). It was suggested that $B$. aphidicola could be considered as an enterobacterial 'gene-order fossil', and that the onset of genomic stasis coincided with the establishment of the symbiosis with aphids. Although no consensus aphid phylogeny has yet been achieved, all of those published show that Aphidinae and Pemphiginae are very divergent lineages (Heie, 1987; Ortiz-Rivas et al, 2003), with estimated divergence times of 84-164 million years (von Dohlen and Moran, 2000). Therefore, considering the divergence time for the $B$. aphidicola strains, the level of genome stability is completely unexpected. On one hand, no horizontal gene transfer insertions can be detected along the $B$. aphidicola genomes, while the incidence in other bacteria reaches $20-30 \%$ in within-species genome comparisons, even over short periods of time, as it is the case of several E. coli strains (Welch et al, 2002). On the other hand, it is remarkable that there is an almost complete absence of inversions and other internal rearrangements in the genome, since inversions are relatively frequent evolutionary events in most of the $\gamma$-proteobacterial lineages (Belda et al, 2005). However,

Table 1 Main genomic features of four analysed B. aphidicola strains and comparison of two selected genome regions

\begin{tabular}{|c|c|c|c|c|c|c|c|c|c|}
\hline \multirow[t]{2}{*}{ Aphid host } & \multirow[t]{2}{*}{ Strain } & \multirow[t]{2}{*}{ Chromosome length (bp) } & \multirow[t]{2}{*}{ Genes } & \multirow[t]{2}{*}{ Accession number } & \multicolumn{5}{|c|}{ rrl-arok and fpr-trxa regions } \\
\hline & & & & & length $(b p)$ & $G+C(\%)$ & ORFS & $R N A S$ & IRs length ${ }^{\mathrm{a}}$ \\
\hline Acyrthosiphum pisum & BAp & 640681 & 608 & NC002528 & 47547 & 29.2 & 60 & 5 & 3126 \\
\hline Schizaphis graminum & $\mathrm{BSg}$ & 641454 & 596 & NC004061 & 46895 & 28.2 & 57 & 5 & 2704 \\
\hline Baizongia pistaciae & $\mathrm{BBp}$ & 615980 & 544 & NC004545 & 44907 & 28.1 & 55 & 5 & 4118 \\
\hline Cinara cedri & $\mathrm{BCC}$ & $\sim 448000$ & Unknown & AY744381; AY744382 & 34576 & 25.4 & 48 & 5 & 2342 \\
\hline
\end{tabular}

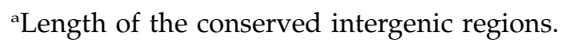


Table 2 Taxonomic status, location and gene order of the leucine cluster and trpEG genes in different strains of $B$. aphidicola from aphids of the family Aphididae

\begin{tabular}{|c|c|c|c|c|c|c|c|c|}
\hline \multicolumn{3}{|c|}{ Aphid host } & \multicolumn{6}{|c|}{ B. aphidicola } \\
\hline \multirow[t]{2}{*}{ Subfamily } & \multirow[t]{2}{*}{ Tribe } & \multirow[t]{2}{*}{ Species } & \multirow[t]{2}{*}{ Strain } & \multicolumn{3}{|c|}{ Leucine cluster } & \multicolumn{2}{|c|}{ trpEG genes } \\
\hline & & & & Plasmid & Chromosome & Accession no. & Location & Accession no. \\
\hline Thelaxinae (T) & & Thelaxes suberi & BTs & $\mathrm{BCDA}$ & & Y11966 & Unknown & Unknown \\
\hline \multirow[t]{2}{*}{ Lachninae (L) } & Eulachnini & Tuberolagnus salignus & BTg & BCDA & & AY546103 & Unknown & Unknown \\
\hline & Lachnini & Cinara cedri & $\mathrm{BCc}$ & $\mathrm{BCDA}$ & & AY438025 & Unknown & Unknown \\
\hline Pterocommatinae (P) & & Pterocomma populeum & $\mathrm{BPp}$ & $\mathrm{ABCD}$ & & AJ006877 & Unknown & Unknown \\
\hline \multirow[t]{4}{*}{ Aphidinae (A) } & Aphidini & Rhopalosiphum padi & $\mathrm{BRp}$ & $\mathrm{ABCD}$ & & X71612 & Plasmid & L43551 \\
\hline & & Schizaphis graminum & $\mathrm{BSg}$ & $\mathrm{ABCD}$ & & AF041836 & Plasmid & Z21938 \\
\hline & Macrosiphini & Acyrthosiphum pisum & BAp & $\mathrm{ABCD}$ & & AJ006878 & Plasmid & AP001070 \\
\hline & & Diuraphis noxia & $\mathrm{BDn}$ & $\mathrm{ABCD}$ & & AF041837 & Plasmid & L46769 \\
\hline \multirow[t]{3}{*}{ Pemphiginae (Pe) } & Pemphigini & Pemphigus spyrothecae & BPs & & $\mathrm{ABCD}(\mathrm{cp})$ & AJ426489; J404864 & Plasmid & AJ012334 \\
\hline & Fordini & Baizongia pistaciae & $\mathrm{BBp}$ & & BCDA (cp) & NC004545 & Chromosome & NC004545 \\
\hline & Eriosomatini & Tetraneura caerulescens & $\mathrm{BTc}$ & & BCDA (cp) & AY375290; Y11974 & Plasmid & AJ012333 \\
\hline Chaitophorinae (C) & & Chaitophorus populeti & $\mathrm{BCp}$ & & $\mathrm{BCDA}$ & AY375291 & Unknown & Unknown \\
\hline
\end{tabular}

(cp), criptic plasmid.

B. aphidicola is not the only lineage with low inversion frequency. The frequency of internal rearrangements in some parts of the genome is extremely low in comparison of Salmonella and Escherichia lineages, which has been related to selection against changes in the position of the genes on the genome (Campo et al, 2004). In the case of $B$. aphidicola, this pattern is associated with an extremely reduced recombination frequency, probably due to the loss of an efficient homologous recombination system (absence of RecA) as well as to the loss of repeated sequences longer than $30 \mathrm{bp}$ in its genome (Klasson and Andersson, 2004).

\section{Gene content varies among lineages}

Despite of the similar genome size of the three sequenced $B$. aphidicola strains, there are some differences in gene content, indicating that independent gene losses have occurred since the Last Common Symbiotic Ancestor of B. aphidicola (LCSA). Since B. aphidicola has maintained the gene order, it was possible to postulate the genome structure of the LCSA of all extant B. aphidicola strains, and to ascertain which specific genes have been lost in the different lineages.

The reconstruction of the minimum gene content of the last common ancestor of $B$. aphidicola and its close relative E. coli (LCA) identified 1818 (Silva et al, 2001) or 2425 genes (Moran and Mira, 2001), depending on the bacterial outgroup being used in the analysis. The complete set of coding genes present in the three sequenced $B$. aphidicola strains is 640 , obtained as the sum of all shared and nonshared genes and pseudogenes, and it represents the most parsimonious reconstruction of the gene content of the LCSA (Silva et al, 2003; van Ham et al, 2003; Gómez-Valero et al, 2004a), indicating that the massive loss of genes occurred in the transition from the LCA to LCSA. The number of genes in the BAp, BSg and BBp genomes is 608, 596 and 544, respectively (Table 1 ). A minimum of 164 independent gene losses have occurred, 96 in the BBp lineage, eight before the split of the two Aphidini, and 24 and 36 in the BAp and BSg lineages, respectively. A set of 139 genes $(22 \%)$ accounts for differences in gene content among the species. It includes pseudogenes, entirely lost genes, and remnant DNA where a gene was located in the past, according to the three postulated steps of genome reduction during $B$. aphidicola genome evolution (Silva et al, 2001). The reason why certain genes are lost (or retained) in some lineages is a matter of debate. The differences in functional gene content between BBp on one hand, and BAp and BSg on the other hand, are substantially greater than the differences between BAp and BSg. When the nature of the lost genes protein products were categorised into the functional Clusters of Orthologous Groups (COGs, Tatusov et al, 1997), we found that the losses embrace all functional categories, although in a different proportion. The majority of the lost genes are involved in metabolic and cellular processes, while the most conserved are genes devoted to information processing (Gómez-Valero et al, 2004a). Moreover, we found that the majority of the losses were not convergent $(80 \%)$, indicating that the differential losses are host-specific, probably due to its particular diet and/or lifecycle. In addition to genes involved in informational processes, the genes involved in the essential amino-acid biosynthetic pathways are also conserved in the three genomes, presumably due to their nutritional role in the symbiosis.

The genome of B. aphidicola $\mathrm{BC}$, from the aphid Cinara cedri (subfamily Lachninae), is currently being obtained in our laboratory. This bacterium possesses the smallest genome reported so far, with an estimated size of $450 \mathrm{~kb}$ (Gil et al, 2002). Our preliminary results showed that the gene order has also been maintained in this strain, confirming the hypothesis that $B$. aphidicola is a geneorder fossil. We have, therefore, been able to perform a detailed analysis of two selected, already sequenced, regions of $\mathrm{BBC}$ which have a very different gene content, and compare them with the corresponding regions in the three previously sequenced B. aphidicola genomes (PérezBrocal et al, 2005). The first region is located between the genes that encode $23 \mathrm{~S}$ ribosomal RNA and shikimate kinase I ( $r r l$-aroK), and mainly contains genes coding for ribosomal proteins. The second region is located between the coding genes for ferredoxine-NADP reductase and thioredoxin $(f p r-\operatorname{tr} x A)$, and it contains mainly genes 
involved in metabolic and cellular processes. The gene order is completely conserved among the four strains in both regions, in spite of a large reduction in gene number (Table 1). In the region rrl-aroK, five genes have been lost with respect to BAp and $\mathrm{BSg}(\arg D, y h f C, \operatorname{yrdC}$, fjpA, and $s m g$ ). Among these genes, $\arg D$ and smg are also absent in BBp. Since the region contains 47 genes in BAp and $\mathrm{BSg}$ (45 in the case of $\mathrm{BBp}$ ), this means that $89 \%$ of the genes have been preserved in BCc. The region fpr-trx $A$ contains 13 genes in BAp and BSg and 10 in BBp. More than half of the genes have been lost in $\mathrm{BC} c$ with respect to BAp and BSg (ynfM, $k d t B$, hem $C$, hemD, yjeA, pitA, yhiQ, yba4 and $u s p A$ ). These results indicate that gene loss has not occurred evenly throughout the genome, but is highly dependent on the nature of the gene function. Thus, the genome reduction in BCc appears mainly to be due to the loss of genes belonging to metabolic and cellular processes, which appear to be nonessential in the protected environment provided by the bacteriocyte, while significantly more genes involved in information processing have been retained in BCC than in the other three strains.

\section{Genome reduction is still an ongoing process}

In bacteria, gene content usually correlates to genome size. The reduction in genome size of intracellular bacteria (symbionts and parasites) is associated with the loss of a great number of genes, probably in response to the new environment, where many molecules can be obtained from the host and do not need to be synthesized. In addition, the endosymbiotic bacteria have lost most of the genes involved in the recombination processes and, consequently, the genome size cannot be increased by acquisition of foreign DNA. It was, therefore, expected that, after the massive gene loss occurred during adaptation to intracellular life from the LCA to the LCSA of B. aphidicola, the preserved genes would be those needed mostly for symbiotic life, with no significant changes in gene content and genome size in different lineages. In fact, the genome sizes of the three sequenced $B$. aphidicola genomes are quite similar (Table 1). However, the PFGE analysis of the chromosome size of nine $B$. aphidicola strains belonging to five aphid subfamilies (Gil et al, 2002) revealed that there is huge size variation among different lineages, ranging from 670 to $450 \mathrm{~kb}$ (Figure 1). B. aphidicola strains from aphids of the subfamilies Chaitophorinae, Thelaxinae and Lachninae have genomes of approximately 450 $550 \mathrm{~kb}$, smaller than the genome of Mycoplasma genitalium, the smallest sequenced bacterial genome $(580 \mathrm{~kb})$ (Fraser et al, 1995). The study also revealed great differences among aphid subfamilies, with the most extreme being the decrease of approximately $200 \mathrm{~kb}$ in $B$. aphidicola from aphids belonging to the subfamily Lachninae, compared to B. aphidicola from the Aphidinae. However, the differences within subfamilies are generally much smaller, being $20 \mathrm{~kb}$ on average (see Figure 1). These results suggest that, although the great majority of genome shrinkage probably occurred in the transition from LCA to LCSA, the different B. aphidicola lineages are still undergoing a reductive process, but at a slower rate. The comparative analysis of two analysed regions across the four $B$. aphidicola strains provides some further clues to address this issue (Pérez-Brocal et al, 2005). At least in

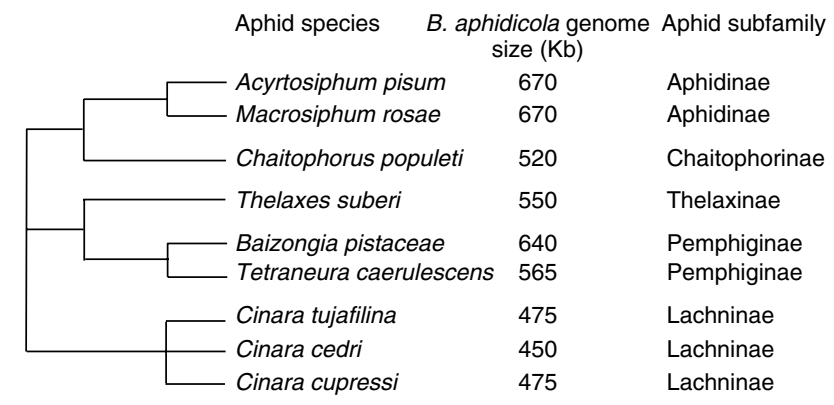

Figure 1 Genome sizes of B. aphidicola from nine aphid species belonging to five aphid subfamilies (Gil et al, 2002). The apparent genome sizes of BAp and BBp strains are slightly higher than those obtained after the sequencing of the complete genome (Shigenobu et al, 2000; van Ham et al, 2003).

these two regions, the reduction process is mainly due to the loss of genes by a process of gene disintegration (Silva et al, 2001), rather than to the shortening of coding regions and/or intergenic regions (IRs). As it can be seen in Table 1, the size of the conserved IRs in the two analysed regions is about the same in BSg and BCc. Gómez-Valero et al (2004a) have estimated that the halflife of a pseudogene in B. aphidicola (ie the period in which half of its nucleotides are lost) is 23.9 million years. It seems then that the size of the different $B$. aphidicola genomes is controlled by restrictions on the loss of function of crucial genes, because when any gene is inactivated, its nucleotides are progressively removed from the genome in a relatively short period of time. Finally, we found that the genome reduction is not random, because more divergent genes are significatively more prone to loss than more conserved ones. More studies should be carried out to test whether there is a correlation between the type of host (ie biological and ecological factors) and B. aphidicola genome size, or whether there are historical contingencies involved in this process. This subject has been approached by genearray hybridisation experiments in other insect models. Using commercial E. coli arrays, a heterologous hybridisation experiment was carried out to infer the genomic composition of Sodalis glossinidius, secondary endosymbiont of the tsetse fly, and SOPE, Sitophilus oryzae primary endosymbiont (Rio et al, 2003). Both bacteria belong to the same clade, and have recently established symbiotic associations, while their hosts belong to different insect orders (Diptera and Coleoptera) and feed on animal blood and stored grain, respectively. The main differences between both genomes appear to reflect differences in the endosymbiotic relationship with their respective hosts, providing insights into the impact of biological and ecological factors on endosymbiont genome evolution.

Are the $B$. aphidicola genomes driven to extinction? It is clear that the loss of some essential metabolic capabilities during the genome reduction process forced intracellular bacteria to depend irreversibly on their host for survival. The question that remains to be solved is whether the final step of the reduction process is the extinction of these endosymbionts. 
Owing to their maternal inheritance, only a small fraction of bacteria pass to the next generation, thus experiencing continuous bottlenecking, and accumulating slightly deleterious mutations in an irreversible way, a phenomenon known as Muller's ratchet (Moran, 1996). The same scenario is true for the oldest known cases of endosymbiosis, which lead to the formation of modern eukaryotic mitochondria and chloroplasts, as a result of symbiogenic events between prokaryotes and primitive eukaryotes around 2000 and 1000 million years ago, respectively (Margulis, 1981). However, in these cases, the establishment of the symbiosis took place at a monocellular stage, and the irreversible bacterial loss of control over their own cellular processes involved the loss of many redundant pathways plus a massive gene transfer from the symbionts to the host nuclear genome, while the existing organelles have retained the functions that make them essential for the survival of the eukaryotic cells. In the present case of bacterial endosymbionts of multicellular organisms, a similar process of horizontal gene transfer from a newly acquired prokaryotic cell to the eukaryotic host must be very difficult. The only reported case has been the transfer of a genome fragment of a Wolbachia endosymbiont to the $\mathrm{X}$ chromosome of its insect host Callosobruchus chinensis (Kondo et al, 2002). In the absence of gene transfer to the host, the loss of essential functions to preserve host fitness might end with the extinction and replacement of these endosymbionts by healthier endosymbiotic bacteria that are present in the same environment. The most direct evidence of secondary symbionts overtaking the role of $B$. aphidicola has been obtained by Koga et al (2003), revealing that, when B. aphidicola was eliminated, the secondary symbionts invaded the bacteriocyte space, establishing a novel endosymbiotic system. Moreover, the infection with secondary symbionts enabled the survival and reproduction of $B$. aphidicola-free aphids. Furthermore, evidence of natural endosymbiotic replacement has been reported recently in some weevil species of the family Dryophtoridae (Lefèvre et al, 2004).

If the prediction is correct, a good aphid-endosymbiont candidate to reach extinction is B. aphidicola BCC, since its genome is so reduced that the impact of Muller's ratchet must be even higher than in the other B. aphidicola genomes. The preserved genes must still be accumulating deleterious mutations, and the compensatory mechanisms, such as the overproduction of GroEL (Fares et al, 2002), would not be enough to maintain the essential functions, which need to be preserved for host fitness. We have recently postulated that the abundant secondary symbionts, which coexist with B. aphidicola $\mathrm{BC} C$ in different bacteriocytes of the aphid, might have taken over at least some of the functions lost by the primary endosymbiont, and might eventually replace it (Gómez-Valero et al, 2004b). However, it still remains to be proven that $B$. aphidicola $\mathrm{BC}$ has lost its ability to provide essential nutrients to the host.

\section{Leucine and tryptophan plasmids go back and forward}

In recent years, genes encoding key enzymes in the pathways leading to tryptophan and leucine biosynthesis (trpEG and leuABCD, respectively) were found to be translocated from the chromosome to plasmids (Lai et al, 1994; Bracho et al, 1995). Amplification of plasmids for essential amino-acid biosynthesis was considered as an indication that $B$. aphidicola is able to overproduce these nutrients for the benefit of its host and, hence, an adaptation to its symbiotic lifestyle. However, the discovery that the main chromosome is present in multiple copies in each cell (Komaki and Ishikawa, 1999), together with the findings that ratios of plasmidborne trpEG and leuABCD copies to chromosomal copies could vary, both within and between species (Thao et al, 1998; Plague et al, 2003), casts doubt on the idea that plasmid location is a means of leucine and tryptophan overproduction. Alternatively, if we consider that the transfer to plasmids occurred when the B. aphidicola genome contained regulatory elements, this could have been a way of skirting genome regulation of leucine and tryptophan biosynthesis by negative feedback, thus allowing a continued supply of these amino acids to the insect, despite the high amino-acid concentration in the bacterial cells.

Extensive studies carried out on the location of the $\operatorname{trp} E G$ and, mainly, on the leuABCD genes in several aphid subfamilies, either on plasmids or on the main chromosome (Lai et al, 1995, 1996; Rouhbakhsh et al, 1996; van Ham et al, 1997, 1999, 2000; Sabater-Muñoz et al, 2002, 2004), revealed a great plasticity throughout $B$. aphidicola evolution, showing that the genomic stasis hypothesis is not supported when plasmids are taken into account. In fact, the evolutionary history of plasmids is puzzling, since not all of the bacterial lineages carry plasmids, and not all plasmids have the same gene content and/or gene order. In addition, when the leucine cluster is located in the chromosome, it is flanked by different genes in different lineages (Sabater-Muñoz et al, 2004), in contrast with the colinearity found in the three sequenced B. aphidicola genomes.

\section{The Leucine cluster}

Up to seven plasmids and four chromosomal locations have been described for the leucine cluster in species belonging to six aphid subfamilies: Thelaxinae, Lachninae, Pterocommatinae, Aphidinae, Pemphiginae and Chaitophorinae (Table 2, Figure 2).

Leucine plasmids, ranging in size from 6.3 to $8.2 \mathrm{~kb}$, are present in the subfamilies Thelaxinae, Lachninae, Pterocommatinae and Aphidinae (van Ham et al, 1997, 2000; Silva et al, 1998; Baumann et al, 1999; Soler et al, 2000). They contain the four structural genes for the synthesis of leucine $(\operatorname{leu} A, \operatorname{leuB}, \mathrm{leu} C$ and leuD) plus one or two genes coding for a plasmid replicase (rep $A 1$ and/ or repA2). Some plasmids also contain the ibp gene, coding for a heat-shock protein, and $y q h \mathrm{~A}$, coding for a putative membrane protein of unknown function. However, the genes $l e u A, B, C$ and $D$ are located on the main chromosome in $B$. aphidicola strains associated with the subfamilies Pemphiginae and Chaitophorinae (SabaterMuñoz et al, 2002, 2004; Van Ham et al, 2003). In addition, all analysed $B$. aphidicola strains from the Pemphiginae possess a cryptic leucine plasmid (cp) of $1.7-2.4 \mathrm{~kb}$ (van Ham et al, 2000) that does not contain the leucine genes.

Contrary to the gene-order conservation observed in the main chromosome, the leucine genes are found in 

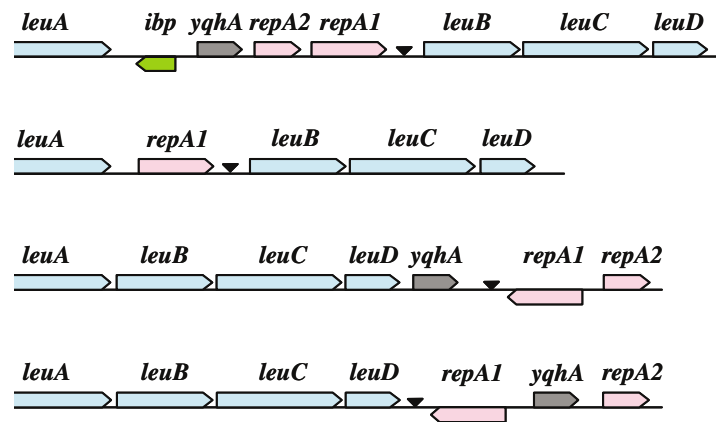
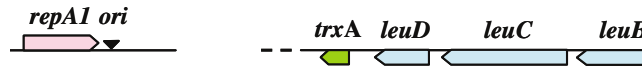

leuA

rep

A repA1 ori

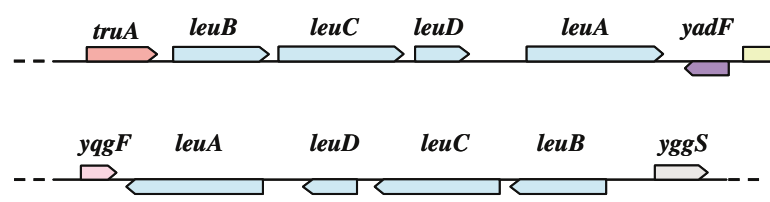

$\operatorname{mrcB}$
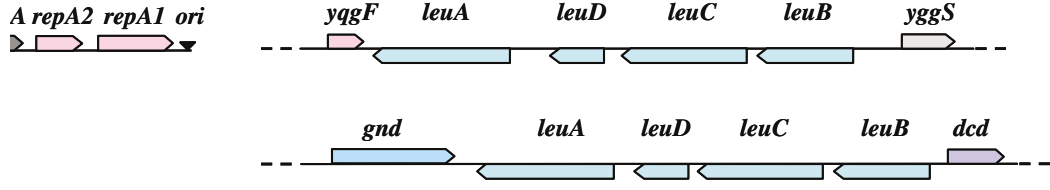

BTs

BTg, BCc

BPp

BRp, BSg, BAp, BDn

BPs

BTc

(T)

BBp

BCp

kb

Figure 2 Structures of the leucine cluster found in the different B. aphidicola strains analysed. (a) Leucine plasmid in species from the subfamilies (T) Thelaxinae, (L) Lachninae, (P) Pterocommatinae and (A) Aphidinae. (b) Chromosomal version of the leucine cluster and flanking regions in species belonging to different tribes of the Pemphiginae (Pe), and in one species of the subfamily Chaitophorinae (C). The structures of the criptic repA plasmid (cp) of the Pemphiginae are also shown. See Table 1 for species code.

different gene orders and flanked by different genes, both when they are located on a plasmid or on the chromosome (Table 2, Figure 2). Bacteria from members of the subfamilies Aphidinae and Pterocommatinae contain the leucine genes on plasmids in the same order as in E. coli (leuABCD), but the companion genes are in a different order, proving the existence of rearrangements, even in those plasmids belonging to endosymbionts of very closely related aphid subfamilies. The other two leucine plasmids present in B. aphidicola strains from aphids of the subfamilies Thelaxinae (BTs) and Lachninae (BTg and $\mathrm{BCC}$ ) show a different order for the leucine genes (leuBCDA). BTg and $\mathrm{BCc}$ contain the simplest leucine plasmids, with only a rep $A$ gene in addition to the four leucine genes. Conversely, the leucine plasmid of BTs contains all the genes found in any of the other seven plasmids, probably representing the original structure of the ancestral plasmid. Finally, there are also some differences among the three cryptic plasmids found in the subfamily Pemphiginae. They are phylogenetically related to the leucine plasmids (van Ham et al, 2000), but instead of having the structural leucine genes, they only code for $i b p$ or $y q h A$ genes, plus one or two replicase genes (Figure 2), depending on the tribe within the subfamily.

In the B. aphidicola strains from aphids of the subfamilies Chaitophorinae and Pemphiginae, the leucine cluster is located at different chromosome positions (Figure 2) (Sabater-Muñoz et al, 2004). In BCp (subfamily
Chaitophorinae), it is flanked by the genes gnd and $d c d$. In the subfamily Pemphiginae, the location of the leucine cluster varies depending on the tribe. In BBp (tribe Fordini), it is flanked by $y q g F$ and $y g g S$, in BPs (tribe Pemphigini), by $\operatorname{tr} x A$ and rep, and in BTc (tribe Eriosomatini), the flanking genes are truA and an ORF homologous to the yadF gene in E. coli (Blattner et al, 1997), upstream of the gene $m r c B$. This ORF is not present in any of the three B. aphidicola sequenced genomes, so it must have been lost in these lineages.

Owing to the extreme gene-order conservation of the B. aphidicola genomes, the variability in the position of the leucine cluster in the chromosome can only be interpreted as resulting from four independent insertions from an ancestral plasmid that contained the leucine genes. The pairs of genes that flank the leucine cluster in each strain (gnd-dcd; yqgF-yggS; trxA-rep and truA-mrcB) are adjacent in BAp, BSg and BCc (unpublished results). Although in the past our research group proposed a chromosomal location for the leucine genes in the LCSA, as in free-living enterobacteria (van Ham et al, 1997), the new findings suggest that a leucine plasmid must have been present in the B. aphidicola LCSA that preceded the diversification of all the endosymbionts, and that the chromosomal location of the leucine genes observed in some $B$. aphidicola strains arose through a back-transfer from the plasmid to the main chromosome. We have performed a phylogenetic reconstruction with the strains shown in Table 2, and with some free-living $\gamma$-proteo- 


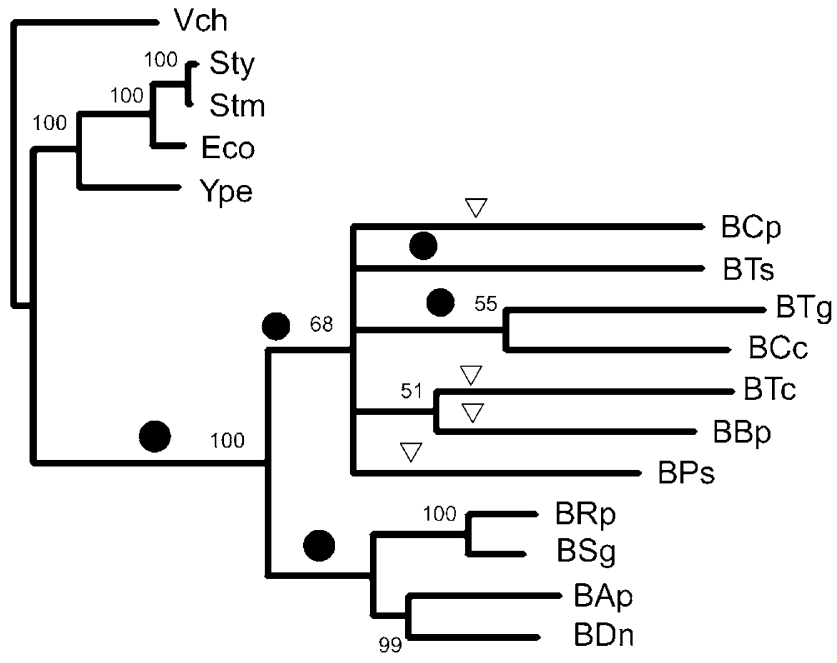

0.1

Figure 3 Phylogenetic tree obtained by maximum likelihood from a concatenated amino-acid alignment of LeuA, LeuB, LeuC and LeuD proteins. Abbreviations of the names of the B. aphidicola strains are shown in Table 2. Free-living bacterial species included are E. coli K12 (Eco, accession number: NC_000913), Salmonella typhimurium LT2 (Stm, acc. number: NC 003197), S. enterica subsp. enterica serovar Typhi str. CT18 (Sty, acc. number: NC_003198), Yersinia pestis CO92 (Ype, acc. number: NC 003143) and the outgroup V. cholerae O1 biovar eltor str. N16961 (Vch, acc. number: NC 002505). The initial alignment was edited using G-BLOCKS (Castresana, 2000) to select the amino-acid positions with the greater phylogenetic information. This edition reduced the alignment to 1075 aminoacid positions ( $67 \%$ of the original one). The phylogenetic tree was inferred by maximum likelihood (ML) combined with the quartet puzzling algorithm implemented in TREEPUZZLE 5.2 (Schmidt et al, 2002). Values at nodes indicate proportion of quarters supporting the corresponding inner branch as determined by the quartet-puzzling method. Close circles indicate the presence of a plasmid in the lineage. Open triangles indicate the insertion of the leucine cluster in the bacterial chromosome.

bacterial species, using a concatenated alignment of the LeuA, LeuB, LeuC and LeuD proteins. The tree topology obtained in this phylogenetic reconstruction (Figure 3 ) agrees with previous ones (even showing the same uncertainties at the subfamily level, Ortiz-Rivas et al, 2003), indicating the common evolutionary origin of the genes located in the plasmids and in the chromosome. A hypothetical scenario of independent back-transfer from an ancestral leucine plasmid to the main chromosome is also shown.

\section{The trpEG genes}

B. aphidicola from aphids of the subfamily Aphidinae and some tribes of the subfamily Pemphiginae also contain tryptophan plasmids (Lai et al, 1996; Rouhbakhsh et al, 1996; van Ham et al, 1999), ranging in size from 3.0 to $12.8 \mathrm{~kb}$, and containing the two first genes of the tryptophan pathway (trpEG) that encode the rate-limiting enzymes for the biosynthesis of tryptophan. The variability in size is mainly due to variation in the number of tandem repeats of these genes or pseudogenes (Lai et al, 1996). The other genes of the tryptophan pathway $(\operatorname{trp} D C(F) B A)$ remain in the main chromosome.
In the subfamily Pemphiginae, where the endosymbionts of the three studied tribes have the leucine cluster located in the chromosome, the trpEG genes are plasmids located in two cases: B. aphidicola BPs (tribe Pemphigini) and BTc (tribe Tetraneurini). However, in BBp and BSc (tribe Fordini), the trpEG genes are chromosomally encoded, thought unlinked to the remaining genes of the pathway (Lai et al, 1995; van Ham et al, 2003) (Table 2; $\mathrm{BSc}$, the endosymbiont of Schlechtendalia chinensis, has not been included in the table because no data for leucine cluster are available). The two trpEG plasmids found in endosymbionts of the Pemphiginae are also structurally different. BTc contains a $3.0 \mathrm{~kb}$ plasmid that carries a single copy of the trpEG and an origin of replication ori3.6. The BPs plasmid contains the genes trpEG and repAC plus a variable number of tandem repeats of a $1.8 \mathrm{~kb}$ unit carrying repAC, trpG and remnants of trpE (van Ham et al, 1999).

In the symbionts of the subfamily Aphidinae, two different trpEG plasmids have been found, which are similar to the plasmids found in the Pemphiginae. All of them hold at least one copy of the trpEG genes plus the ori3.6 region, and resemble the plasmid found in BTc. Some of them include a variable number of the trpEG genes or pseudogenes (Lai et al, 1996; Rouhbakhsh et al, 1996). The plasmid of B. aphidicola BRm (from Ropalosiphum maidis) also contains the gene rep $A C$, and therefore it is similar to the plasmid found in BPs. In BRp, repAC appears as a pseudogene. These findings suggest a common ancestor for all these tryptophan plasmids, which probably contained a repAC gene that has been lost and replaced by the ori3.6 region in some modern lineages (van Ham et al, 1999).

Phylogenetic analyses carried out by van Ham et al (1999) with TrpE and TrpG sequences from several Aphidinae endosymbionts and the Pemphiginae endosymbionts BTc, BPs and BSc showed that the plasmidborne sequences of BTC and BPs are more closely related to the chromosomal trpEG sequence of $\mathrm{BSc}$ than to the Aphidinae plasmid-borne sequences. Although only trpEG genes from $B$. aphidicola belonging to two aphid subfamilies have been studied at present (Aphidinae and Pemphiginae), these results support a common origin of the genes located either in a plasmid or in the chromosome, as for the leucine genes.

\section{A proposed evolutionary scenario}

Since the LCSA, the evolutionary dynamics have been very different for the $B$. aphidicola chromosome and plasmids. The extraordinary gene-order conservation of the main chromosome contrasts starkly with the versatility of leucine and tryptophan plasmids, regarding gene content and location, in the different B. aphidicola lineages.

We propose that the leucine cluster was transferred from the main chromosome to a repA plasmid at some stage between the LCA and the LCSA of B. aphidicola. Since then, at least four independent back-transfers have occurred throughout B. aphidicola evolution (Figure 3). The ancestral LCSA leucine plasmid would contain the four leucine genes and at least one $\operatorname{rep} A$, plus two additional genes, $i b p$ and $y q h A$, resembling the BTs leucine plasmid (Figure 2). Regarding the trpEG genes, 
although the analysis was carried out in B. aphidicola from only two aphid subfamilies, an evolutionary scenario similar to the one we propose for the leucine genes has also been postulated. The transfer to a plasmid carrying a repA/C-like replicon would have occurred in the ancestor of all present-day $B$. aphidicola strains, followed by independent events of replicon replacement, and back-transfer of trpEG to the chromosome in different lineages (van Ham et al, 1999). Thus, the ancestral LCSA tryptophan plasmid would contain the gene $\operatorname{rep} A C$, and at least one copy of $\operatorname{trpEG}$.

The transfer of the bacterial rate-limiting genes for the biosynthesis of leucine and tryptophan from the main chromosome to plasmids must have been a key event for the successful adaptation of the ancestor of modern aphids to unbalanced diets, by avoiding the regulatory feedback control of both operons. However, extant aphid species are extremely diverse biologically and ecologically, and some of them may require relatively little leucine or tryptophan provisioning from their endosymbionts. During the coevolution of $B$. aphidicola and the aphids, it must have been advantageous to adjust the amino-acid biosynthesis to the insects' needs. A first step in the regulation of the levels of such amino acids might be related to the control of plasmid copy number (Thao et al, 1998; Plague et al, 2003), as well as the number of tandem repeats of the plasmid-borne genes and its later pseudogenisation (in the case of the trpEG genes). At some point, the loss of $B$. aphidicola genes involved in the control of DNA replication and segregation led to polyploidy of the bacterial cells. In addition, other regulatory elements upstream to structural genes for the biosynthetic enzymes were also lost or altered (Moran et al, 2003). In this new scenario, the backtransfer of the plasmid-borne genes to the chromosome might have been advantageous, which would explain why, in several B. aphidicola lineages, these genes are present in the main chromosome. At least four independent events of back-transfer have been identified for the leucine cluster. Since $\operatorname{rec} A$ is absent from the three sequenced $B$. aphidicola genomes, we have postulated that the insertions would have been mediated by the rec $B C D$ system that has been retained (Sabater-Muñoz et al, 2004). This same system could be responsible for the gene-order rearrangements found in the different leucine plasmids. Finally, the cryptic plasmid found in the $B$. aphidicola associated with the Pemphiginae would be a remnant of the back-transfer of the leucine genes to the chromosome, instead of a cryptic rep $A$ plasmid present in the LCSA, as previously proposed (van Ham et al, 2000). Probably, these plasmids are retained because they contain essential genes (ibp or $y q h A$ ) that were not transferred to the chromosome in the recombination event.

\section{Acknowledgements}

We thank Dr C Biémont for his kind invitation to write this review and to an anonymous reviewer for his/her valuable comments. This research was funded by grants Grupos03/204 from Govern Valencià, and BFM200300305 from Ministerio de Ciencia y Tecnología (MCyT) to A Moya. RG is a recipient of a contract in the Ramon $y$ Cajal Program from the MCyT, Spain.

\section{References}

Akman L, Yamashita A, Watanabe H, Oshima K, Shiba T, Hattori $\mathrm{M}$ et al (2002). Genome sequence of the endocellular obligate symbiont of tsetse flies, Wigglesworthia glossinidia. Nat Genet 32: 402-407.

Andersson SG, Kurland CG (1998). Reductive evolution of resident genomes. Trends Microbiol 6: 263-268.

Baumann L, Baumann P, Moran NA, Sandström J, Thao ML (1999). Genetic characterization of plasmids containing genes encoding enzymes of leucine biosynthesis in endosymbionts (Buchnera) of aphids. J Mol Evol 48: 77-85.

Baumann P, Moran NA, Baumann L (2000). Bacteriocyteassociated endosymbionts of insect. In: Dworkin $\mathrm{M}$ (ed) The Prokaryotes. Springer-Verlag: New York, pp 1-67 (Online). http:/ /link.springer.de/link/service/books/10125/.

Belda E, Moya A, Silva FJ (2005). Genome rearrangement distances and gene order phylogeny in $\gamma$-proteobacteria. Mol Biol Evol 22: 1456-1467.

Blattner FR, Plunkett III G, Bloch CA, Perna NT, Burland V, Riley $\mathrm{M}$ et al (1997). The complete genome sequence of Escherichia coli K-12. Science 227: 1453-1474.

Bracho AM, Martínez-Torres D, Moya A, Latorre A (1995). Discovery and molecular characterization of a plasmid localized in Buchnera sp. bacterial endosymbionts of the aphid Rhopalosiphum padi. J Mol Evol 41: 67-73.

Buchner P (1965). Endosymbiosis of Animals with plants Microorganisms. Interscience Publishers, Inc: New York.

Campo N, Días MJ, Daveran-Mingot ML, Ritzenthaler P, Le Bourgeois P (2004). Chromosomal constraints in Grampositive bacteria revealed by artificial inversions. Mol Microbiol 51: 511-522.

Castresana J (2000). Selection of conserved blocks from multiple alignments for their use in phylogenetic analysis. Mol Biol Evol 17: 540-552.

Clark M, Moran NA, Baumann P (1999). Sequence evolution in bacterial endosymbionts having extreme base composition. Mol Biol Evol 16: 1586-1598.

Douglas AE (1998). Nutritional interactions in insect-microbial symbioses: aphids and their symbiotic bacteria Buchnera. Ann Rev Entomol 43: 17-37.

Fares MA, Ruiz-Gonzalez MX, Moya A, Elena SF, Barrio E (2002). GroEL buffers against deleterious mutations. Nature 417: 398.

Fraser CM, Gocayne JD, White O, Adams MD, Clayton RA, Fleischmann RD et al (1995). The minimal gene complement of Mycoplasma genitalium. Science 270: 397-402.

Gil R, Sabater-Muñoz B, Latorre A, Silva FJ, Moya A (2002). Extreme genome reduction in Buchnera spp.: toward the minimal genome needed for symbiotic life. Proc Nat Acad Sci USA 99: 4454-4458.

Gil R, Silva FJ, Zientz E, Delmotte F, González-Candelas F, Latorre A et al (2003). The genome sequence of Blochmannia floridanus: comparative analysis of reduced genomes. Proc Nat Acad Sci USA 100: 9388-9393.

Gómez-Valero L, Latorre A, Silva FJ (2004a). The evolutionary fate of nonfunctional DNA in the bacterial endosymbiont Buchnera aphidicola. Mol Biol Evol 21: 2172-2181.

Gómez-Valero L, Soriano-Navarro M, Pérez-Brocal V, Heddi A, Moya A, García-Verdugo JM et al (2004b). Coexistence of Wolbachia with Buchnera aphidicola and a secondary symbiont in the aphid Cinara cedri. J Bacteriol 186: 6626-6633.

Heidelberg JF, Eisen JA, Nelson WC, Clayton RA, Gwinn ML, Dodson RJ et al (2000). DNA sequence of both chromosomes of the cholera pathogen Vibrio cholerae. Nature 406: 477-483.

Heie OE (1987). Paleontology and Phylogeny. In: Minks AK, Harrewijn P (eds) Aphids. Their Biology, Natural Enemies and Control. Elsevier: Amsterdam. pp 367-391.

Klasson L, Andersson SGE (2004). Evolution of minimal-genesets in host-dependent bacteria. Trends Genet 12: 37-43.

Koga R, Tsuchida T, Fukatsu T (2003). Changing partners in an obligate symbiosis: a facultative endosymbiont can compen- 
sate for loss of the essential endosymbiont Buchnera in an aphid. Proc R Soc Lond B 270: 2543-2550.

Komaki K, Ishikawa H (1999). Intracellular bacterial symbionts of aphids posses many genomic copies per bacterium. J Mol Evol 48: 717-722.

Kondo N, Nikoh N, Ijichi N, Shimada M, Fukatsu T (2002). Genome fragment of Wolbachia endosymbiont transferred to X chromosome of host insect. Proc Nat Acad Sci USA 99: 14280-14285.

Lai CY, Baumann L, Baumann P (1994). Amplification of trpEG: adaptation of Buchnera aphidicola to an endosymbiotic association with aphids. Proc Nat Acad Sci USA 91: 3819-3823.

Lai CY, Baumann P, Moran NA (1995). Genetics of the tryptophan biosynthetic pathway of the prokaryotic endosymbiont (Buchnera) of the aphid Schlechtendalia chinensis. Insect Mol Biol 4: 47-59.

Lai CY, Baumann P, Moran NA (1996). The endosymbiont (Buchnera sp.) of the aphid Diruaphis noxia contains plasmids consisting of trpEG and tandem repeats of trpEG pseudogenes. Appl Environ Microbiol 62: 332-339.

Latorre A, Gil R, Silva FJ, Martínez-Torres D, Moya A (2003). Tempo and mode of genome evolution in endosymbiotic bacteria of insects: the case of Buchnera aphidicola. Symbiosis 34: 301-316.

Lefèvre C, Charles H, Vallier A, Delobel B, Farell B, Heddi A (2004). Endosymbiont phylogenies in the Dryophtoridae weevils: evidence for bacterial replacement. Mol Biol Evol 21: 965-973.

Margulis L (1981). Symbiosis in Cell Evolution. Freeman: San Francisco.

Moran NA (1996). Accelerated evolution and Muller's ratchet endosymbiotic bacteria. Proc Nat Acad Sci USA 93: 2873-2878.

Moran NA (2002). Microbial minimalism: genome reduction in bacterial pathogens. Cell 108: 583-586.

Moran NA, Mira A (2001). The process of genome shrinkage in the obligate symbiont Buchnera aphidicola. Genome Biol 2 RESEARCH 0054

Moran NA, Munson MA, Baumann P, Ishikawa H (1993). A molecular clock in endosymbiotic bacteria is calibrated using the insect hosts. Proc R Soc Lond B 253: 167-171.

Moran NA, Plague GR, Sandström JP, Wilcox JL (2003). A genomic perspective on nutrients provisioning by bacterial symbionts of insects. Proc Nat Acad Sci USA 100: 14543-14548.

Moya A, Latorre A, Sabater B, Silva FJ (2002). Comparative molecular evolution of primary (Buchnera) and putative secondary endosymbionts of aphids based on two proteincoding genes. J Mol Evol 54: 127-137.

Munson MA, Baumann P, Kinsey MG (1991). Buchnera gen. Nov. and Buchnera aphidicola sp. Nov., a taxon consisting of the mycetocyte-associated, primary endosymbionts of aphids. Int J Syst Bacteriol 41: 566-568.

Ortiz-Rivas B, Moya A, Martínez-Torres D (2003). Molecular systematic of aphids (Homoptera: Aphididae): new insights from the long-wave length Opsina gene. Mol Phyl Evol 30: 24-37.

Pérez-Brocal V, Latorre A, Gil R, Moya A (2005). Comparative analysis of two genomic regions among four strains of Buchnera aphidicola, primary endosymbiont of aphids. Gene 345: 73-80.

Plague GR, Dale C, Moran NA (2003). Low and homogeneous copy number of plasmid-borne symbiont genes affecting host nutrition in Buchnera aphidicola of the aphid Uroleucon ambrosiae. Mol Ecol 12: 1095-1100.

Rio VM, Lefèvre C, Heddi A, Aksoy S (2003). Comparative genomics of insect-symbiotic bacteria: influence of host environment on microbial genome composition. Appl Environ Microbiol 69: 6825-6832.

Rouhbakhsh D, Lai CY, von Dohlen C, Clark MA, Baumann L, Baumann P et al (1996). The tryptophan biosynthetic path- way of aphids endosymbionts (Buchnera): genetics and evolution of plasmid-associated anthranilate synthase (trpEG) within the Aphididae. J Mol Evol 42: 414-421.

Sabater-Muñoz B, Gómez-Valero L, van Ham RCHJ, Silva FJ, Latorre A (2002). Molecular characterization of the leucine cluster in Buchnera sp. Strain PSY, a primary endosymbiont of the aphid Pemphigus spyrothecae. Appl Environt Microbiol 68: $2572-2575$.

Sabater-Muñoz B, van Ham RCHJ, Moya A, Silva FJ, Latorre A (2004). Evolution of the leucine gene cluster in Buchnera aphidicola: insights from chromosomal version of the cluster. J Bacteriol 186: 2646-2654.

Sasaki T, Hayashi H, Ishikawa H (1991). Growth and reproduction of symbiotic and aposymbiotic pea aphids, Acyrtosiphon pisum, maintained on artificial diets. I Insect Physiol 37: 85-92.

Schmidt HA, Strimmer K, Vingron M, von Haeseler A (2002). TREE-PUZZLE: maximum likelihood phylogenetic analysis using quartets and parallel computing. Bioinformatics 18 : 502-504.

Shigenobu S, Watanabe H, Hattori M, Ishikawa H (2000). Genome sequence of the endocellular bacterial symbionts of aphids Buchnera sp. APS. Nature 407: 81-86.

Silva FJ, Latorre A, Moya A (2001). Genome size reduction through multiple events of gene disintegration in Buchnera APS. Trends Genet 17: 615-618.

Silva FJ, Latorre A, Moya A (2003). Why are the genomes of endosymbiotic bacteria so stable? Trends Genet 19: 176-180.

Silva FJ, van Ham RCHJ, Sabater-Muñoz B, Latorre A (1998). Structure and evolution of the leucine plasmids carried by the endosymbionts (Buchnera aphidicola) from aphids of the family Aphididae. FEMS Microbiol Lett 168: 43-49.

Soler T, Latorre A, Sabater B, Silva FJ (2000). Molecular characterization of the leucine plasmid from Buchnera aphidicola, primary endosymbionts of the aphid Acyrtosiphon pisum. Curr Microbiol 40: 264-268.

Tamas I, Klasson L, Canback B, Naslund AK, Eriksson AS, Wernegreen JJ et al (2002). 50 million years of genomic stasis in endosymbiotic bacteria. Science 296: 2376-2379.

Tatusov RL, Koonin EV, Lipman DJ (1997). A genomic perspective on protein families. Science 278: 631-637.

Thao ML, Baumann L, Bauman P, Moran NA (1998). Endosymbionts (Buchnera) from the aphids Schizaphis graminum and Diuraphis noxia have different copy numbers of the plasmid containing the leucine biosynthetic genes. Curr Microbiol 36: 238-240.

van Ham R, Kamerbeek J, Palacios C, Rausell C, Abascal F, Bastolla $U$ et al (2003). Reductive genome evolution in Buchnera aphidicola. Proc Nat Acad Sci USA 100: 581-586.

van Ham RCHJ, Gonzalez-Candelas F, Silva FJ, Sabater B, Moya A, Latorre A (2000). Postsymbiotic plasmid acquisition and evolution of the repA1-replicon in Buchnera aphidicola. Proc Nat Acad Sci USA 97: 10855-10860.

van Ham RCHJ, Martínez-Torres D, Moya A, Latorre A (1999). Plasmid-encoded anthranilate synthase (trpEG) in Buchnera aphidicola from aphids of the family Pemphigidae. Appl Environ Microbiol 65: 117-125.

van Ham RCHJ, Moya A, Latorre A (1997). Putative evolutionary origin of plasmids carrying the genes involved in leucine biosynthesis in Buchnera aphidicola (endosymbiont of aphids). I Bacteriol 179: 4768-4777.

von Dohlen CD, Moran NA (2000). Mitochondrial ribosomal sequences and fossils support a rapid radiation of aphids in the Cretaceous and multiple origins of a complex life cycle. Biol J Linn Soc 71: 689-717.

Welch RA, Burland V, Plunkett III G, Redford P, Roesch P, Rasko $\mathrm{D}$ et al (2002). Extensive mosaic structure revealed by the genome sequence of uropthogenic Escherichia coli. Proc Nat Acad Sci USA 99: 17020-17024. 